\title{
A General Mathematical Formulation for the Determination of Differential Leakage Factors in Electrical Machines with Symmetrical and Asymmetrical Full or Dead-coil Multi-phase Windings
}

\author{
M. Caruso, A. O. Di Tommaso, F. Genduso, R. Miceli, Member, IEEE, and G. Ricco Galluzzo \\ Department of Energy, Information Engineering and Mathematical Models, University of Palermo, \\ Viale delle Scienze, Building nr. 9, 90128 - Palermo, Italy
}

\begin{abstract}
This paper presents a simple and general mathematical formulation for the determination of the differential leakage factor for both symmetrical and asymmetrical full and deadcoil windings of electrical machines. The method can be applied to all multi-phase windings and considers Görges polygons in conjunction with masses geometry in order to find an easy and affordable way to compute the differential leakage factor, avoiding the adoption of traditional methods that refer to the Ossanna's infinite series, which has to be obviously truncated under the bound of a predetermined accuracy. Moreover, the method described in this paper allows the easy determination of both the minimum and maximum values of the differential leakage factor, as well as its average value and the time trend. The proposed method, which does not require infinite series, is validated by means of several examples in order to practically demonstrate the effectiveness and the easiness of application of this procedure.
\end{abstract}

Index Terms-Differential leakage factor, dead-coil windings, asymmetrical windings, concentrated windings, multi-phase windings, Görges polygon, moment of inertia.

\section{List OF MAIN SYMBOLS}

$N \quad$ Number of slots;

$p \quad$ Number of pole pairs;

$m \quad$ Number of phases;

$\mathbb{N} \quad$ The set of integer numbers;

$\mathbb{G} \quad$ The set of the even numbers;

$\mathbb{U}$ The set of the odd numbers;

$\gamma \quad$ Number of coils per phase;

$g \quad$ Number of phasors with different phases;

$Q \quad$ Number of slots per pole and per phase;

$t \quad$ Time variable;

$k_{w \nu} \quad$ Winding factor for the $\nu$-th harmonic order;

$I, I_{x}$ Phase current (RMS and peak value).

\section{INTRODUCTION}

Nowadays, most of the design procedures for electrical machines are based on the fundamental wave theory, which is focused on the achievement of a sinusoidal distribution of the air-gap flux density with the lowest possible harmonic content [1]-[6]. However, depending on specific targets, other design criteria can be adopted, such as, for instance, the optimization of concentrated windings [7], the structural mass minimization of direct-drive generators [8] or the use of no rare-earth magnet machines [9]. In any case, the differential leakage factor is an important parameter that can be considered as a measure for the distortion rate that affects the magneto motive force (MMF) distribution in the air-gap of an electrical machine. Moreover, it has been demonstrated that this parameter depends on the harmonic distribution of winding factors and, consequently, on the type of winding pattern chosen for a particular machine [2], [10]-[18].

Therefore, an exact knowledge of the differential leakage factor (from which average torque, parasitic torques and forces, copper and eddy current losses can be determined [17], [19]) is a significant aid to design also fractional-slot single and double layer windings with low torque ripple, from symmetrical to asymmetrical configurations (at least if they have a low degree of unbalance) [19], [20], even in the case of the so called "dead coil windings" (dead coil windings contain one or more empty slots in single-layer or non wound coils in double-layer windings). Regarding asymmetrical windings, the formula for the determination of differential leakage factors becomes more complex with respect to symmetrical ones and only few authors dealt with this particular topic [21], [22].

Asymmetrical windings are commonly adopted in threephase, pole-changing induction machines with speed ratios equal to 2:3 [22], 4:6 [21] and so on, in machines with Pole Amplitude Modulation (PAM) [2], or when the use of the same stator laminates for more slot/pole number combinations [23] is required. Other possible applications can be associated to the rewinding of an old machine for a new synchronous speed [24] (e.g. 3000 or 1500 to $1000 \mathrm{rpm}$ at $f=50 \mathrm{~Hz}$ [22]), or the optimization of the motor performances by reducing the effects of slot harmonics [25], [26]. However, the adoption of slightly asymmetrical windings can also be an interesting choice during the design stage of electrical machines. In fact, the use of this type of winding configuration in several case studies can even provide benefits and advantages if compared with symmetrical topologies, significantly increasing the possibility of choice in terms of slot/pole number combinations and improving the industrial automated manufacturability of all electrical machines, even in the case of automotive applications. 
For instance, either a 24 slots/6 poles or a 30 slots/6 poles threephase machine is composed by slightly asymmetrical windings; however, due to the light degree of unbalance, the electrical and magnetic properties can be comparable to the symmetrical ones [20]. In fact, it can be stated that even windings designed to be perfectly symmetrical present slight asymmetries due to unavoidable manufacturing tolerances.

In this context, this paper presents a simple and effective method for the computation of the differential leakage factor suitable for all types of winding configurations: symmetrical, asymmetrical, including dead-coil ones. This method is based on the construction of Görges polygons. The adoption of this procedure allows the computation of only the winding factor of the working harmonic (the one having $p$ pole pairs), together with the moment of inertia matrix of the Görges polygons associated to the winding, avoiding traditional procedures that involve very complex and troublesome computations. Moreover, the proposed method allows the easy determination of both the minimum and maximum values of the differential leakage factor, as well as the average value and its trend over time. By adopting this fast procedure, the design space could be improved by performing several variations of a base-winding configuration in order to optimize its performances (i.e. varying the number of slots or pole pairs or phases by using zone widening or imbrication techniques, shortened pitch coils, etc). Therefore, an online re-assembly of the base winding and a fast procedure for the calculation of the related leakage factor could significantly reduce the time of computing and it can immediately determine the performance improvement.

It has to be highlighted that the main paper in literature dealing with this topic was presented by the Authors in [27]. However, that method was directed only for asymmetrical windings without dead-coils and it was not demonstrated its applicability towards reduced poly-phase systems (such as dual symmetrical six-phase windings [28]). In fact, the procedure presented in [27] has been significantly modified in order to extend the method to all possible types of winding configurations. Thus, this paper proposes a simple and general method that can be applied to all symmetrical and asymmetrical windings both distributed and concentrated, even for normal and reduced multi-phase systems, including those having a number of phases that is a power of two.

More in detail, this work is structured as follows: Section II provides some definitions regarding the symmetry conditions of winding configurations, whereas Section III describes the equations proposed in the recent scientific literature for the computation of the differential leakage factor. Section IV discusses about the method based on the Görges polygon presented by the Authors and some examples are given in Section $\mathrm{V}$ in order to demonstrate the versatility and the ease of application of the proposed procedure. Finally, Section VI proposes a comparison between the results obtained with finite element analysis and those achieved by means of the proposed method.

\section{Symmetry CONDITIONS FOR Winding DESIGN}

This Section provides a possible general definition for both symmetrical and asymmetrical windings.
For an electrical machine with $N$ slots, $m$ phases, $n_{l}$ layers, and $p$ pole pairs, the winding symmetry can be evaluated by using the factors $\gamma$ and $g$ defined as [29]:

$$
\gamma=n_{l} \frac{N-\eta}{2 m}
$$

$$
g= \begin{cases}\frac{N}{m r} & \text { for normal and non - reduced systems } \\ \frac{N}{2 m r} & \text { for reduced systems, }\end{cases}
$$

where $\eta$ represents the number of empty slots (for a dead-coil winding) in the case of single layer windings and the number of unwound coils in the case of double layer windings and:

$$
r=\operatorname{gcd}(N, p)
$$

is the greatest common divider between $N$ and $p$.

The winding symmetry is defined as follows:

$$
\begin{cases}\gamma \in \mathbb{N} & \text { for coil windings } \\ \gamma \in\{\mathbb{N}, \mathbb{N} / 2\} & \text { for bar windings, }\end{cases}
$$

and

$$
\begin{cases}g \in \mathbb{N} & \text { for symmetrical windings } \\ g \notin \mathbb{N} & \text { for asymmetrical windings, }\end{cases}
$$

If conditions (4) and the first of (5) are satisfied, the winding is symmetrical; otherwise, it is asymmetrical. It has to be highlighted that condition 4 can be always verified by adequately choosing $\eta$. Thus, possible asymmetries can be brought back to condition 5, which refers to the phase-shift angles between the phases (for more detail, see also [29]). These conditions of symmetry can be applied to both normal (otherwise named radially-symmetrical) systems, defined as multi-phase systems having a no-loaded neutral point [28], and reduced systems.

\section{DifFEREntial LEAKAGE FACtor: A Literature REVIEW}

The leakage factor is defined with the following general formula [1], [2], [11], [13]-[15], [17], [30]-[32]:

$$
\sigma_{0}=\frac{W_{\delta}-W_{p}}{W_{p}}=\frac{W_{\delta}}{W_{p}}-1
$$

where $W_{\delta}$ is the whole magnetic energy stored in the airgap and $W_{p}$ is the air-gap magnetic energy contribution limited to the working harmonic. By manipulating (6), the following equation can be derived:

$$
\sigma_{0}=\left(\frac{p}{k_{w p}}\right)^{2} \sum_{\nu=1, \nu \neq p}^{+\infty}\left(\frac{k_{w \nu}}{\nu}\right)^{2}
$$

where $k_{w p}$ is the winding factor at the working harmonic $(\nu=$ p) [10], [13].

Nevertheless, equation (7) is limited only to symmetrical windings, even if it can be applied for many winding configurations, including integer and fractional types. In addition, the summation (known as the Ossanna's series [31]) has the form of an infinite series that slowly converges [2], [11], [13] and its computation, in many cases, results particularly troublesome. 
Huang [11] and Kron [31] proposed two similar procedures for the calculation of (7) in a closed form. However, both methodologies require the determination of the winding factors within at least one period, which leads to complex computations.

With regards to asymmetrical windings, which present different winding factors for each phase, Heller et al. [12] demonstrated the following expression of the differential leakage, which was also described and used by Cistelecan et al. [22], [32]:

$$
\sigma_{0}(t)=\sigma_{0 m}+\sigma_{0 v} \cdot \cos (2 \omega t+\varphi),
$$

where

$$
\begin{gathered}
\sigma_{0 m}=\left(\frac{p}{k_{w p}}\right)^{2} \sum_{\nu=1, \nu \neq p}^{+\infty}\left[\left(\frac{k_{w d \nu}}{\nu}\right)^{2}+\left(\frac{k_{w i \nu}}{\nu}\right)^{2}\right], \\
\sigma_{0 v}=2\left(\frac{p}{k_{w p}}\right)^{2} \sum_{\nu=1, \nu \neq p}^{+\infty} \frac{k_{w d \nu} \cdot k_{w i \nu}}{\nu^{2}}
\end{gathered}
$$

$\nu$ is the harmonic order, $\omega=2 \pi f$ and $\varphi$ is a generic angular phase. It can be noticed from (8) that the leakage factor is time-dependent and it is composed by 2 components: (9), which is constant in time, and (10), which is the amplitude of a periodic function of twice the supply frequency. These two terms have the same behavior as Ossanna's series with a very weak convergence. The alternative formulas, presented in the next section, will provide a calculation method overcoming this problem. It will also be demonstrated that, by applying the Gorges polygon, it is possible to determine both $\sigma_{0 m}$ and $\sigma_{0 v}$ without the need of complex equations.

\section{Determination of the Differential Leakage FACTOR AND THE MOMENT OF INERTIA OF GÖRGES POLYGONS}

The proposed analysis is carried out by considering the following assumptions:

1) The magnetic voltage drop is confined within the air-gap, which implies that the flux density is radially directed.

2) The air-gap is considered with constant length, neglecting the effects of proximity of the slots. Therefore, the magnetic energy is stored only in the air-gap and not in the slots.

3) The winding phase currents are considered as a symmetric multi-phase system with sinusoidal distribution in time.

4) The unipolar and the circulation flux density components are neglected.

Therefore, the magnetic energy stored in the air-gap can be expressed as follows:

$$
W_{\delta}=\frac{1}{2} \int_{V} h(\xi) \cdot b(\xi) d V=\frac{\mu_{0} \delta " l_{i}}{2} \int_{0}^{2 p \tau} h^{2}(\xi) d \xi
$$

where $d V=\delta " l_{i} d \xi$ is the elementary air-gap volume,

$$
h(\xi)=\frac{b(\xi)}{\mu_{0}}=\frac{v(\xi)}{\delta^{\prime \prime}},
$$

$b(\xi)$ and $h(\xi)$ are the spatial distributions of the air-gap flux density and the air-gap magnetic field intensity, respectively, $\xi$

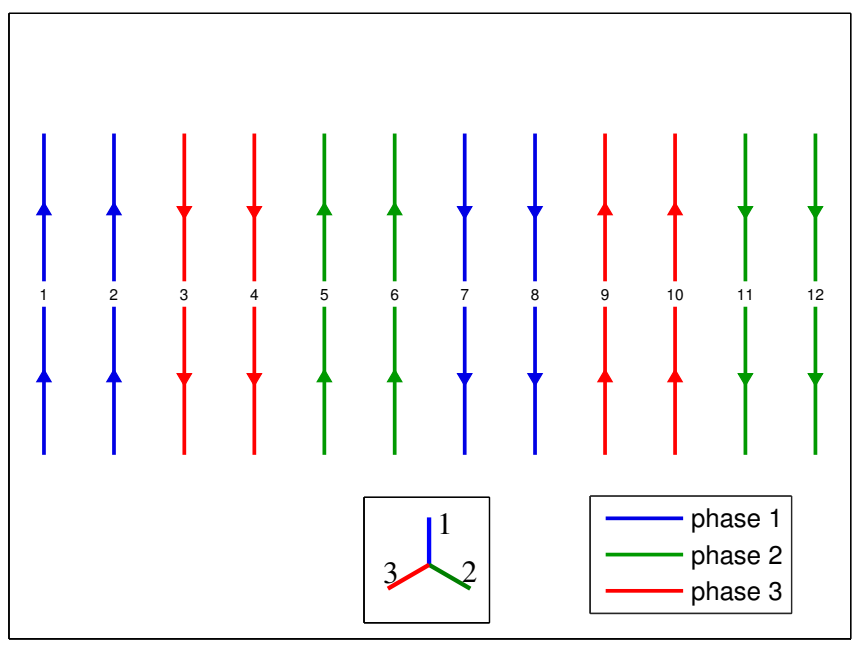

Figure 1. Example of plane scheme from which the Görges polygon can be derived.

is the spatial coordinate along the air-gap, $\mu_{0}$ is the permeability of vacuum and air, $\delta "=k_{C} k_{\text {sat }} \delta$ is the fictive air-gap length that accounts the slot opening $\left(k_{C}\right.$ is the Carter factor [28], [33], [34]) and the saturation of the magnetic circuit $\left(k_{s a t}\right.$ is the saturation factor [2], [28]), $l_{i}$ is the ideal axial length of the machine and $\tau$ is its pole pitch. In (12) $v(\xi)$ is the MMF spatial distribution along the air-gap.

By considering a discrete MMF air-gap distribution, (11) can be expressed as

$$
W_{\delta}=\frac{\mu_{0} l_{i} p \tau}{\delta^{\prime \prime}} \cdot \frac{1}{N} \sum_{i=1}^{N} \nu_{i}^{2},
$$

where $v_{i}$ is the instantaneous value of air-gap MMF at a generic $i$-th slot. Moreover, the energy $W_{p}$ is determined through equation

$$
W_{p}=\frac{\mu_{0} l_{i}}{2 \delta^{\prime \prime}} \int_{0}^{2 p \tau} \nu_{p}^{2}(\xi) d \xi=\frac{\mu_{0} l_{i} p \tau}{2 \delta^{\prime \prime}} \cdot V_{p}^{2},
$$

where

$$
v_{p}(\xi)=V_{p} \sin \left(\frac{\pi}{\tau} \xi\right) .
$$

Therefore, the differential leakage factor can be determined with the following formula:

$$
\sigma_{0}=\frac{W_{\delta}}{W_{p}}-1=\frac{2}{V_{p}^{2}} \cdot \frac{1}{N} \sum_{i=1}^{N} \nu_{i}^{2}-1
$$

where

$$
V_{p}=\sqrt{2} \frac{m w k_{w p}}{\pi p} I=\frac{m w k_{w p}}{\pi p} I_{x}
$$

and $w$ is number of series connected coil turns per phase. The obtained formula can be applied to both symmetrical and asymmetrical winding configurations; however, for asymmetrical types, the direct-sequence component of $k_{w p}$, namely $k_{w p d}$, which is derived from the Fortescue's theorem [35], is considered. 


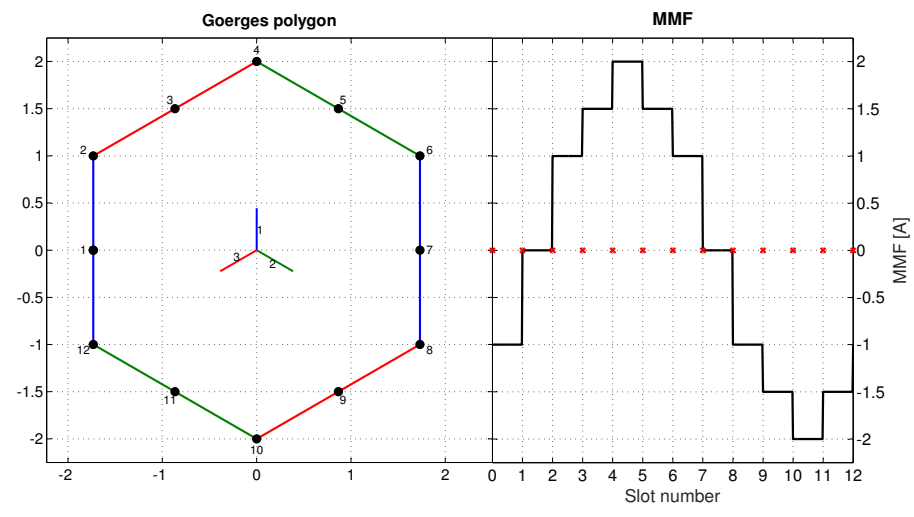

Figure 2. Example of Görges polygon construction and related MMF waveform for an electrical machine with $N=12, p=1$ and $m=3$.

The construction of the Görges polygon can be clarified by the following example, which considers a 3-phase machine with $p=1$ pole pair and equipped with a symmetrical, single-layer winding located into $N=12$ slots. The procedure starts from the knowledge of the current distribution along the stator periphery, as shown in Fig. 1, which depicts the planar scheme of the coil sides located in the slots, each of them already characterized by both their phase belonging (colors) and sign (up or down arrows). By assuming a symmetrical three-phase current system represented by the colored phasors in the same Figure, it can be noticed that slot nr. 1 is characterized by a positive current belonging to phase nr.1 (blue up arrow). Therefore, an oriented segment (vector) with the same orientation of phase 1 vector is drawn (segment $\overline{12-1}$ in blue color), as shown in Fig. 2. The point of application of this first oriented segment can be chosen at own free will. The same current flows also in slot nr. 2 (see Fig. 1) and, therefore, a second segment in-phase with phase 1 vector is plotted (segment $\overline{1-2}$ in blue color), with its point of application coincident with the end of the first vector (point nr. 1 in Fig. 2). Slot nr. 3 belongs to phase 3 and it is characterized by a negative current (down arrow); therefore, a vector opposite to phase 3 vector (segment $\overline{2-3}$ in red color) is plotted with its point of application at point nr. 2, and so on. By adding all the characterized vectors in the graph of Fig. 2, the Görges polygon can be closed. Finally, the coordinates of the center of mass are calculated by means of the following formulas

$$
x_{M}=\frac{1}{N} \sum_{i=1}^{N} x_{i} \text { and } y_{M}=\frac{1}{N} \sum_{i=1}^{N} y_{i},
$$

and the whole polygon is moved by $x_{M}$ and $y_{M}$ in their respective directions in order to ensure that the flux density field maintains the solenoidal property. For double layer windings the same procedure is applied by considering the resultant MMF in each slot, due to the presence of two coil sides.

The projections of the vertexes on the vertical axis $y$ coincide with the instantaneous values of the air-gap MMF distribution at each stator slot, as shown in Fig. 2, which depicts the Görges polygon together with the MMF spatial distribution for the proposed example. The polygon and the related MMF are determined considering a 3-phase symmetrical current system at the time instant when the current flowing in phase 1 is at its maximum instantaneous value. It can also be noticed that the distances of the vertexes of the polygon with respect to the horizontal axes $x$ represent the MMF value related to each slot (see Fig. 2). During time the Görges polygon rotates counterclockwise at the angular speed $\omega=2 \pi f$ and, meanwhile, the MMF changes its shape, traveling in the right direction.

By taking into account the procedures previously described (see also [27], [36] and [24]) and by inspection of Fig. 2, it can be seen that the summation term $\sum_{i=1}^{N} \nu_{i}^{2}$ contained in (15) is equal to the moment of inertia, calculated with respect to the horizontal $x$ axis, of a system of $N$ unitary concentrated masses displaced at the $N$ vertexes of the Görges polygon.

In case of symmetrical winding configurations, the Görges polygon has a gyroscopic geometry, which leads to the fact that the moment of inertia computed with respect to a generic axis passing through the center of mass assumes a constant value, independently from the axis orientation: this means that symmetrical windings are characterized by a constant differential leakage factor. On the contrary, in case of asymmetrical winding configurations, the Görges polygon is not, in general, a gyroscope and, therefore, the moment of inertia varies dependently on the direction of the axis taken into account for its computation. In this case, it can be demonstrated that formula (8) is valid. Therefore, it is necessary to determine the maximum and minimum values of the moment of inertia during an electrical period (corresponding to one revolution of the Görges polygon about its center of mass). The steps to develop formulas to characterize the minimum and maximum moments of inertia are reported below.

For this purpose, the "time axis" $u$ is here defined as an axis passing through the origin $O$, having a generic direction and rotating clockwise at angular speed $\omega=2 \pi f$. In this case, the Görges polygon is considered stationary and the projections of its vertexes on the time axis provide the instantaneous values of the air-gap MMF at each slot. The moment of inertia $J_{u}$ with respect to the time axis $u$ is defined as:

$$
J_{u}=\boldsymbol{u}^{T} \cdot \boldsymbol{J} \cdot \boldsymbol{u}
$$

where

$$
\boldsymbol{u}=\left[\begin{array}{ll}
\cos (\theta) & \sin (\theta)
\end{array}\right]^{T}
$$

is the unitary vector of axis $u(\theta=\omega t$ is the angle of the time axis with respect to the horizontal $x$ axis, which depends on time and on the supply frequency), whereas

$$
\boldsymbol{J}=\left[\begin{array}{cc}
J_{x x} & -J_{x y} \\
-J_{x y} & J_{y y}
\end{array}\right]
$$

is the moment of inertia matrix. Moreover,

$$
J_{x x}=\sum_{i=1}^{N} y_{i}^{2}
$$

is the moment of inertia of the mass system with respect to the $x$ axis (the principal moment of inertia with respect to $x$ ),

$$
J_{y y}=\sum_{i=1}^{N} x_{i}^{2}
$$


is the moment of inertia of the mass system with respect to the $y$ axis (the principal moment of inertia with respect to $y$ ) and

$$
J_{x y}=J_{y x}=\sum_{i=1}^{N} x_{i} \cdot y_{i}
$$

are the products of inertia of the same mass system. When the masses constitute a gyroscopic system $J_{x x}=J_{y y}$, the products of inertia vanish, i.e. $J_{x y}=J_{y x}=0$, and matrix $\boldsymbol{J}$ becomes diagonal. Here $x_{i}$ and $y_{i}$ represent the coordinates of the generic $i$-th vertex of the polygon in the $x-y$ plane, respectively.

For the computation of the moment of inertia of Görges polygons for either symmetrical or asymmetrical multi-phase windings, even with dead coils, the following formulas, derived by carrying out the scalar products in (17), can then be adopted:

$$
J_{u}=J_{x x} \cos ^{2}(\theta)+J_{y y} \sin ^{2}(\theta)-2 J_{x y} \sin (\theta) \cos (\theta),
$$

which can be expressed in a more compact form as

$$
J_{u}=\frac{J_{p}}{2}+\sqrt{\left(\frac{J_{x x}-J_{y y}}{2}\right)^{2}+J_{x y}^{2}} \cdot \cos (2 \theta+\varphi),
$$

by introducing the polar moment of inertia

$$
J_{p}=J_{x x}+J_{y y}=\sum_{i=1}^{N}\left(y_{i}^{2}+x_{i}^{2}\right)
$$

and the phase

$$
\varphi=\operatorname{sign}\left(I_{x y}\right) \cdot \arccos \frac{J_{x x}-J_{y y}}{2 \sqrt{\left(\frac{J_{x x}-J_{y y}}{2}\right)^{2}+J_{x y}^{2}}} .
$$

Now, it can be demonstrated, following the steps outlined in [27], that the relation between the moment of inertia of the Görges polygon and the differential leakage factor $\sigma_{0}(t)$ is:

$$
\sigma_{0}(t)=\frac{2 J_{u}(t)}{N \cdot V_{p}^{2}}-1=\frac{J_{u}(t)}{p m Q \cdot V_{p}^{2}}-1,
$$

where $V_{p}$ is the maximum value of the MMF working harmonic distribution in the air-gap and $Q=N / 2 p m$ is the number of slots per pole and per phase.

An alternative expression of the differential leakage factor can be obtained by introducing the concept of "radius of gyration". In this case

$$
\sigma_{0}(t)=\frac{\rho^{2}(t)}{V_{p}^{2}}-1
$$

where

$$
\rho(t)=\sqrt{\frac{J_{u}(t)}{p m Q}}=\sqrt{\frac{2}{N} J_{u}(t)}
$$

is the radius of gyration referred to the Görges polygon.

By comparing (24) with (8), it is possible to deduce the following relations:

$$
\sigma_{0 m}=\frac{J_{p}}{N \cdot V_{p}^{2}}-1
$$

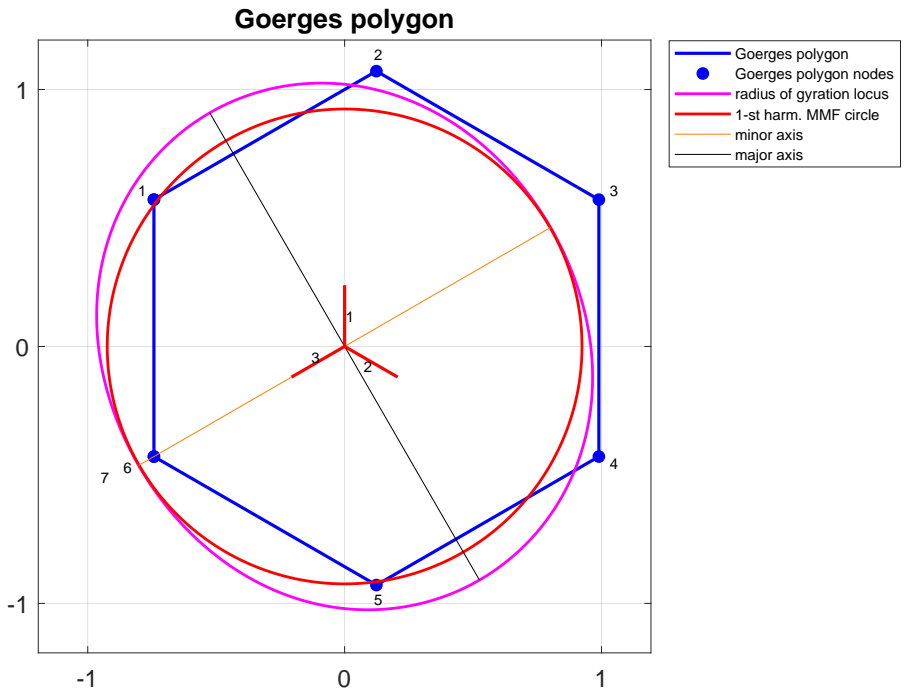

Figure 3. Görges polygon of a simple single-layer winding with $N=7$, $m=3, p=1, \eta=1, Q=1+1 / 6$ and $q=1$.

$$
\begin{gathered}
\sigma_{0 v}=\frac{2}{N \cdot V_{p}^{2}} \sqrt{\left(\frac{J_{x x}-J_{y y}}{2}\right)^{2}+J_{x y}^{2}} \\
\sigma_{0 \min }=\sigma_{0 m}-\sigma_{0 v}
\end{gathered}
$$

and

$$
\sigma_{0 \max }=\sigma_{0 m}+\sigma_{0 v},
$$

These parameters are adopted in order to determine the average, the minimum and the maximum differential leakage factors, respectively.

To clarify the role of empty slots a simple example, referred to a single-layer asymmetrical winding (this winding is considered only for demonstrative purpose) with one empty slot, is considered, whose Görges polygon is shown in Fig. 3. The winding is characterized by a number of wound slots per pole and per phase $q=N-\eta / 2 p m=1$ and by a number of slots per pole and per phase $Q=N / 2 p m=1+1 / 6$.

Even if the slot nr. 7 is assumed to be empty, the vertex nr. 7 in the Görges polygon is also taken into account for the computation of its moment of inertia, because this slot is affected by the same MMF value of slot nr. 6. In this case, two unitary masses must be considered as concentrated in the same vertex. Therefore, it can be generally stated that the contribution of an empty $i$-th slot will be equal to the contribution of the slot $i-1$. As a consequence, even though the polygon still maintains a symmetrical form, its center of mass does not coincide with the polygon center. The computation of the principals moments of inertia for the proposed example, for sake of clarity, are reported in Appendix.

In this case, such as in almost all general cases of asymmetrical windings, the polygon is not a gyroscope and, therefore, the moment of inertia will vary during a revolution around the origin, as shown in Fig. 3, where the closed magenta curve represents the radius of gyration locus. This curve has a minor axis and a major one (the two eigenvectors of matrix $\boldsymbol{J}$ ) that identify the directions and the values of the minimum and 
the maximum moment of inertia, respectively. Thus, during a complete revolution of the the Görges polygon (i.e. during an electrical period), its moment of inertia assumes twice the maximum and twice the minimum values. The red circle represents the locus of the working harmonic of the air-gap spatial MMF distribution (here $\nu=p=1$ ).

It has to be highlighted that the proposed method considerably reduces the computational effort to determine the leakage factor for asymmetrical windings with respect to other existing approaches, such as [11] and [12]. In fact, the proposed method allows the determination of both minimum and maximum values of $\sigma_{0}$ with only one configuration of the air-gap MMF for any possible winding topology. On the contrary, equation (15) requires at least two steps of computation related to two airgap space distribution of the MMF, whereas [12] requires the solution of a series with infinite terms, which leads to an approximated solution due to truncation, and the burden of calculation for both direct and inverse winding factor harmonic components. Moreover, the methodology presented in [11] requires the knowledge of at least $(N-1) / 2$ harmonic winding factors, whereas the proposed method needs the computation of only the winding factor at the working harmonic, or its direct component in case of asymmetrical windings.

\section{EXAMPLES}

This Section presents some examples in order to demonstrate the easiness of computation of the differential leakage factor by means of the proposed procedure, which can be applied for both symmetrical and asymmetrical windings, even in the case of reduced, non-reduced, distributed or concentrated ones. The pictures have been carried out by means of the ACWindSoft 6.3 software [37], [38]. The examples referring to asymmetrical windings will consider only slight unbalances, i.e with a degree of unbalance (D.U.\%) equal to:

$$
\text { D.U. } \%=\frac{k_{w p_{i}}}{k_{w p_{d}}} \cdot 100 \leq 5 \%,
$$

where $k_{w p_{d}}$ and $k_{w p_{i}}$ are the direct and inverse components of the winding factor at the working harmonic, respectively, which can be computed by applying Fortescue's theorem to the winding factors.

In the following, for all representations of the Görges polygon, the magenta curves represent the radius of gyration, whereas the red ones represent the locus of the air-gap spatial MMF distribution working harmonic. Moreover, for each of the proposed examples, the results in terms of differential leakage factor have been compared with those obtained by means of (15). However, it has to be highlighted that, in all cases involving asymmetrical windings, this equation allows the determination of only one instantaneous (actual) value of the leakage factor, i.e for a specified assignment of the phase currents, avoiding the possibility of predetermining exactly its maximum and minimum values. On the contrary, as previously mentioned, the method described in this work allows the easy determination of both the minimum and maximum values of $\sigma_{0}$, as well as its average value and its trend over time by virtue of (26) or (27), which is strictly dependent on the instantaneous values of the supply currents that determine the spatial position of the Görges polygon. In any case, by referring to (15), the instantaneous values of the leakage factor for an entire electrical cycle have been computed for any example in which the Görges Polygon is not gyroscopic. It has to be highlighted that, for all the other examples, the leakage factor is a constant value for the entire cycle, leading to an equality in terms of minimum, maximum, average and actual values.

\section{A. Symmetrical Windings}

Example 1. A seven-phase symmetrical double-layer winding, located into $N=49$ slots, with $p=1$ pole pairs and $q=3+1 / 2$ $(\gamma=7 \in \mathbb{N}$ and $g=7 \in \mathbb{N}$ ), whose winding diagram is depicted in Fig. 4a, is considered as a first validation example. The related Görges polygon assumes the geometry represented in Fig. 4b, whereas the resulting differential leakage factor is equal to $\sigma_{0}=0.14 \%$, which is equal to the computed one by means of (15) $\sigma_{0 \text { (1lay)act }}=0.14 \%$.

\section{B. Asymmetrical Windings}

Example 2. The first example taken into account for asymmetrical windings is a 3-phase machine equipped with a double-layer, fractional configuration with $N=31, p=3, q=(N-\eta) / 2 p m=$ $1+2 / 3, Q=N / 2 p m=1+13 / 18$ and $\eta=1(\gamma=10 \in \mathbb{N}$ and $g=10+1 / 3 \notin \mathbb{N}$ ). Here $\eta=1$ means that a coil is missing, namely the coil whose positive side is located into slot nr. 11 . The missing coil ensures that the first condition of (4) is verified. Figs. $5 \mathrm{a}$ and $5 \mathrm{~b}$ represent the winding connection scheme and the Görges polygon, respectively, by which it is possible to see clearly the effect of the winding asymmetry on the shape of the Görges polygon. In addition, Figure 5b shows (together with the Görges polygon) the locus representing the radius of gyration (in magenta color) and the polar circle of gyration (red color) related to the working harmonic (here $\nu=p=3$ ). The resulting minimum and maximum differential leakage factor are respectively: $\sigma_{0 \min }=4.18 \%$ and $\sigma_{0 \max }=8.92 \%$, whereas the unbalance factor of the winding is D.U.\% $=1.96 \%$. The actual leakage factor computed by means (15) is $\sigma_{0 a c t}=5.3717 \%$. The variability of $\sigma_{0 a c t}$ over an entire electrical cycle has been determined and plotted in Fig. 6.

Example 3. An asymmetrical, double-layer fractional full-coil three-phase winding is considered with $N=30, p=3$, $q=(N-\eta) / 2 p m=1+2 / 3, Q=N / 2 p m=1+2 / 3$, $\eta=0(\gamma=10 \in \mathbb{N}$ and $g=3+1 / 3 \notin \mathbb{N})$. Figs. $7 \mathrm{a}$ and $7 \mathrm{~b}$ represent the winding connection scheme and the Görges polygon, respectively. The resulting differential leakage factor is: $\sigma_{0 \min }=\sigma_{0 \max }=\sigma_{0}=8.04 \%$ (actual leakage is $\sigma_{\text {0actmin }}=\sigma_{\text {0actmax }}=\sigma_{0 a c t}=8.04 \%$ ), whereas the unbalance factor of the winding is $D . U . \%=1.31 \%$. In this case, although the winding results asymmetrical, the Görges polygon is symmetrical, the locus of the radius of gyration becomes a circle and the differential leakage factor $\sigma_{0}$ is constant. Therefore, it can be stated that the choice of an asymmetrical winding with a symmetrical Görges polygon can be even a favorable option during the design stage of an electrical machine in order to considerably decrease the differential leakage factor.

Example 4. An asymmetrical 5-phase winding is considered with the following characteristics: $N=31$ slots, $p=14$, $m=5, \eta=1$. The machine is equipped with an asymmetrical 


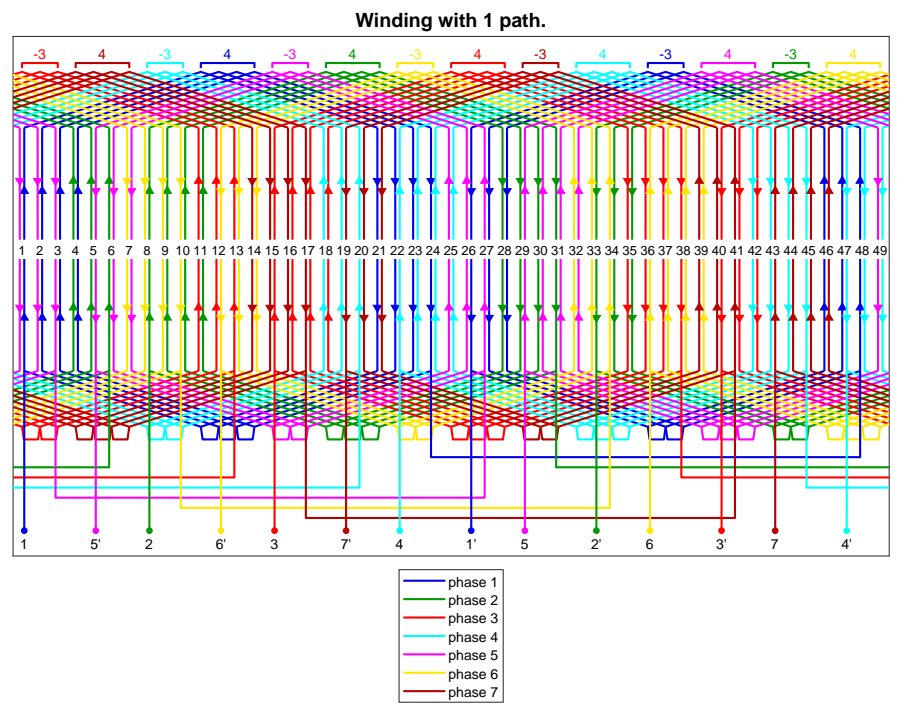

(a) Winding scheme.

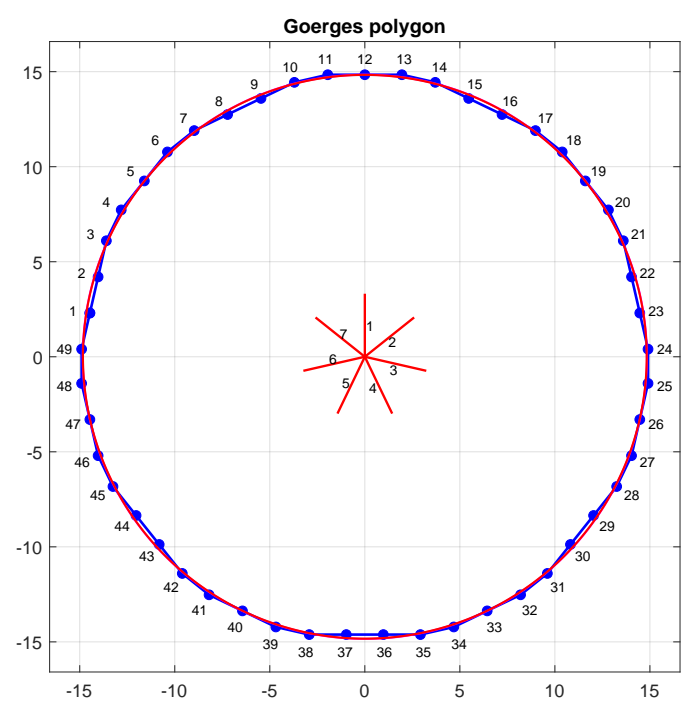

(b) Görges polygon.

Figure 4. 7-phase machine with symmetrical double-layer winding: $N=49, p=1$.

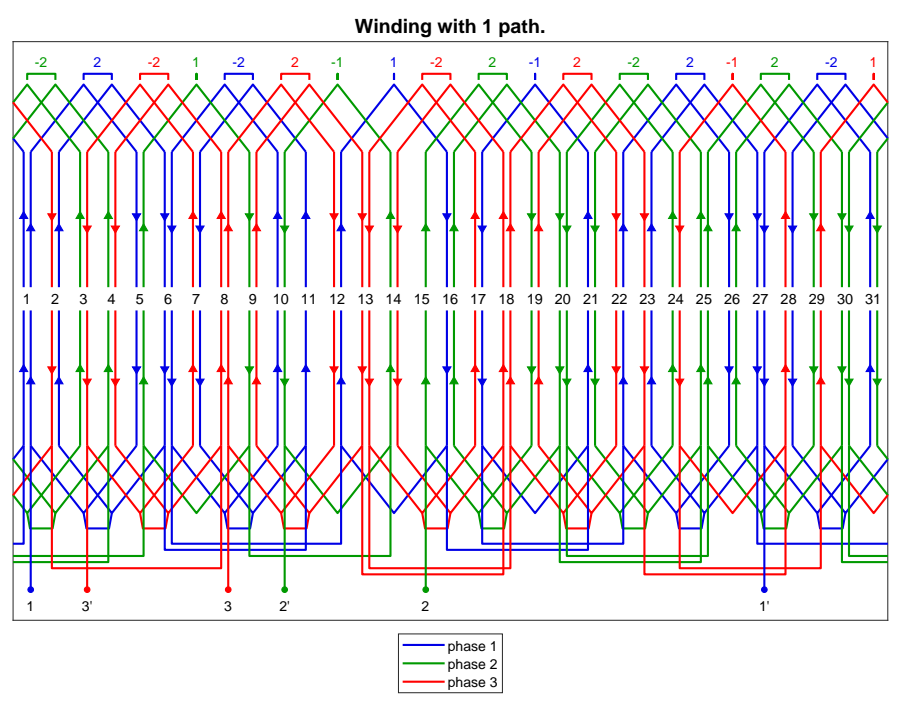

(a) Winding scheme.

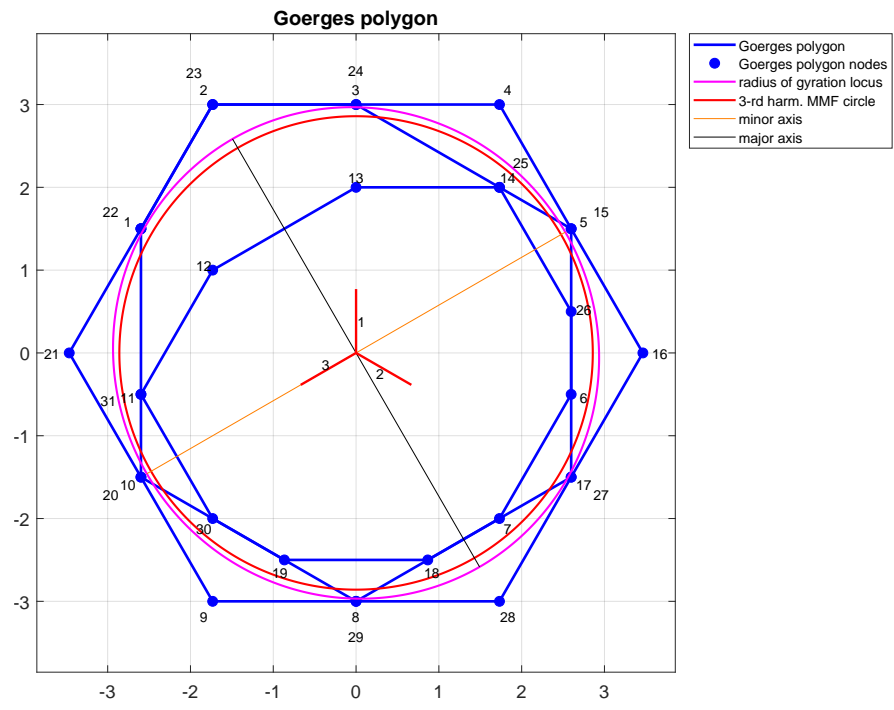

(b) Görges polygon.

Figure 5. 3-phase machine with asymmetrical double-layer fractional winding: $N=31, p=3, \eta=1$.

double-layer concentrated and non overlapping winding with $Q=31 / 140, q=3 / 14(\gamma=6 \in \mathbb{N}$ and $g=6+1 / 5 \notin \mathbb{N})$. The winding diagram and the Görges polygon are represented in Figs. 8a and 8b, respectively. It should be noted that also in this case the polygon is symmetric and the resulting differential leakage factor maintains a constant value in time, equal to $\sigma_{0 \min }=\sigma_{0 \max }=\sigma_{0}=119.56 \%$, which is equal to the value obtained through (15) $\sigma_{0 \text { actmin }}=\sigma_{0 a c t \max }=\sigma_{0 a c t}=$ $119.56 \%$. This high differential leakage factor is typical for concentrated windings, in which the spatial distribution of the MMF at the air-gap is highly distorted.

Moreover, the coil whose sides should be located in slots nr. 12 and 13 is missing, due to condition (4). The unbalance factor of the winding is relatively low and equal to D.U.\% $=1.74 \%$.

Finally, the winding factors at the working harmonic for all the proposed examples are summarized in Table I and computed by means of the procedure described in [29]. In case of asymmetrical configurations, the Fortescue's theorem has been applied in order to determine $k_{w p_{d}}$ and $k_{w p_{i}}$ [35].

\section{FEM VALIDATION}

In order to strengthen the validity of the method described in the previous Sections, the Finite Element Analysis is taken into account. More specifically, an induction machine composed by a six-phase, double-layer, reduced (otherwise named dual-threephase) winding with $N=36$ and $p=2(\gamma=6 \in \mathbb{N}$ and $g=3 \in \mathbb{N}$ ) has been considered as a validation example.

The FEMM4.2 software has been used in order to create the related model of the motor, whose winding scheme is reported in Fig. 9a. This configuration is partially symmetrical, due to the fact that, even if the two groups of three-phase systems composing the winding are symmetrical, the phaseshift between the two groups is $27.6^{\circ}$ (slightly different from the 
Table I

WINDING FACTORS FOR ALL OF THE PROPOSED EXAMPLES

\begin{tabular}{c|c||c|c|c|c|c}
\multicolumn{2}{c|}{} & $N 49, p 1, m 7$ & $N 36, p 2, m 6$ & $N 31, p 3, m 3$ & $N 30, p 3, m 3$ & $N 31, p 14, m 5$ \\
\hline \hline \multirow{2}{*}{ Phase nr.1 } & Ampl. & 0.9508 & 0.9715 & 0.9582 & 0.9064 & 0.9737 \\
\cline { 2 - 7 } & ph. arg. & $0^{\circ}$ & $0^{\circ}$ & 0 & $0^{\circ}$ & 0 \\
\hline \multirow{2}{*}{ Phase nr. 2} & Ampl. & 0.9508 & 0.9715 & 0.9582 & 0.9064 & 0.9737 \\
\cline { 2 - 7 } & ph. arg. & $51.4^{\circ}$ & $120^{\circ}$ & $116.1^{\circ}$ & $115.4^{\circ}$ & $69.7^{\circ}$ \\
\hline \multirow{2}{*}{ Phase nr. 3 } & Ampl. & 0.9508 & 0.9715 & 0.9582 & 0.8784 & 0.9737 \\
\cline { 2 - 7 } & ph. arg. & $102.9^{\circ}$ & $240^{\circ}$ & $238.1^{\circ}$ & $237.7^{\circ}$ & $139.4^{\circ}$ \\
\hline \multirow{2}{*}{ Phase nr. 4 } & Ampl. & 0.9508 & 0.9715 & - & - & 0.9737 \\
\cline { 2 - 7 } & ph. arg. & $154.3^{\circ}$ & $26.7^{\circ}$ & - & - & $214.8^{\circ}$ \\
\hline \multirow{2}{*}{ Phase nr. 5 } & Ampl. & 0.9508 & 0.9715 & - & - & -9737 \\
\cline { 2 - 7 } & ph. arg. & $205.7^{\circ}$ & $146.7^{\circ}$ & - & - & - \\
\hline \multirow{2}{*}{ Phase nr. 6 } & Ampl. & 0.9508 & 0.9715 & - & - & - \\
\cline { 2 - 7 } & ph. arg. & $257.1^{\circ}$ & $266.7^{\circ}$ & - & - & - \\
\hline \multirow{2}{*}{ Phase nr. 7 } & Ampl. & 0.9508 & - & - & - & - \\
\cline { 2 - 7 } & ph. arg. & $308.6^{\circ}$ & - & 0.9578 & 0.8966 & 0.9733 \\
\hline \hline
\end{tabular}

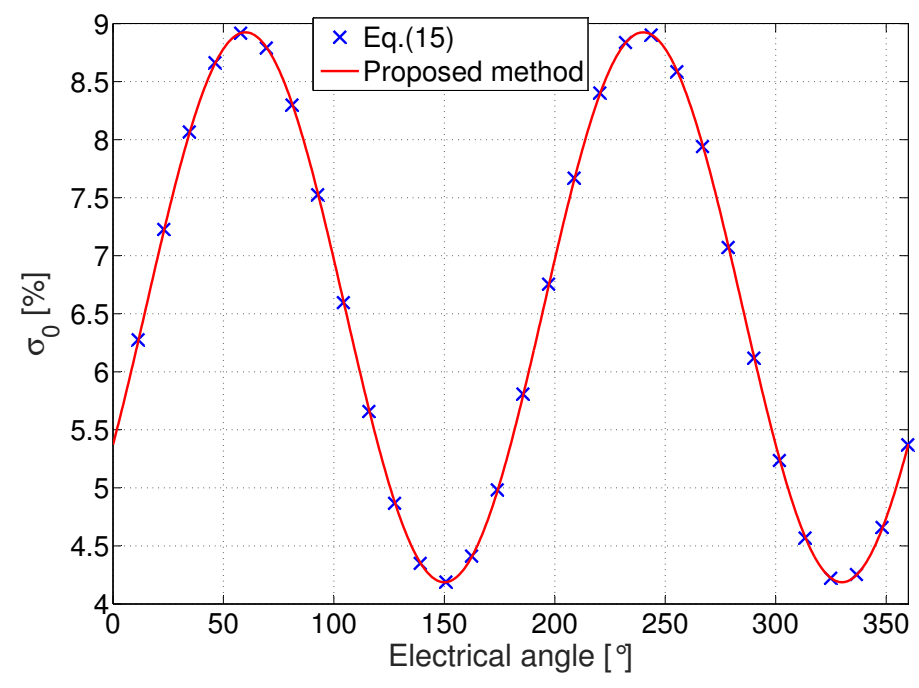

Figure 6. Variability of $\sigma_{0}$ over an entire electrical period.

symmetry angle of $30^{\circ}$ ), which leads to a very slight asymmetry. Fig. 9b shows the simulation results in terms of flux density plot derived from the FEM analysis, whereas the Görges polygon related to this example is depicted in Fig. 9c.

The differential leakage computed by means of the Görges polygon varies between a minimum value $\sigma_{0 \text { min }}=0.99 \%$ and a maximum value of $\sigma_{0 \max }=1.01 \%$, which are really close to each other. The actual leakage factor computed with (15) is $\sigma_{0 a c t}=1.00 \%$. The variability of $\sigma_{0 a c t}$ over an entire electrical cycle can be appreciated in Fig. 9d (crossed points), in which the maximum and minimum values are equal to those obtained with the proposed method (red trend). Moreover, by processing the data acquired from the FEM analysis concerning the values of air-gap flux density field over an entire electrical cycle and by applying (13), (14) and (15), the values of $\sigma_{0 F E M}$ have been computed and compared with those obtained by means of the proposed procedure and by (15). This comparison can be appreciated in Fig. 9d, which confirms the validity of the method proposed by the Authors.

\section{CONCLUSIONS}

The method presented in this paper allows a significant simplification regarding the computation of the Ossanna's series in order to obtain the exact solution for all possible topologies of windings, even for dead-coil or reduced ones. In fact, the introduced formulas allow to reduce this series to a sum of a finite number of terms, requiring, furthermore, only the calculation of the winding factor at the working harmonic. The method is very flexible: it allows the easy determination of both the minimum and maximum values of the differential leakage factor, as well as its average value and the time trend. Moreover, it avoids the burden of calculation of both direct and inverse winding factor harmonic components and it can be easily applied by using a computer, for any number of phases and independently from the winding configuration, such as non-reduced, reduced, full-coil, dead-coil, asymmetrical and symmetrical ones.

\section{APPENDIX}

By considering the example reported in Fig. 3, for which the winding scheme is plotted in Fig. 10, the principal moments of inertia and the products of inertia can be calculated by means of (20), (21) and (22), respectively:

$$
\begin{aligned}
J_{x x}= & 0.7423^{2}+2 \cdot 0.1237^{2}+2 \cdot 0.9897^{2}+2 \cdot 0.7423^{2}= \\
= & 3.6426 \mathrm{~A}^{2}, \\
& J_{y y}=0.5714^{2}+1.0714^{2}+0.5714^{2}+0.9286^{2}+ \\
\quad & +3 \cdot 0.4286^{2}=3.2143 \mathrm{~A}^{2}, \\
& \\
J_{x y}= & -0.7423 \cdot 0.5714+0.1237 \cdot 1.0714+ \\
& +0.9897 \cdot 0.5714-0.9897 \cdot 0.4286+ \\
- & 0.1237 \cdot 0.9286+2 \cdot 0.7423 \cdot 0.4286=0.3711 \mathrm{~A}^{2},
\end{aligned}
$$

whereas (16) gives, considering a peak current value $I_{x}=1$ A,

$$
V_{p}=\frac{3 \cdot 1 \cdot 0.9677}{\pi \cdot 1} \cdot 1=0.9241 \mathrm{~A} .
$$

Finally, the average value and the amplitude of the variable part of the differential leakage factor are given, respectively, by: 


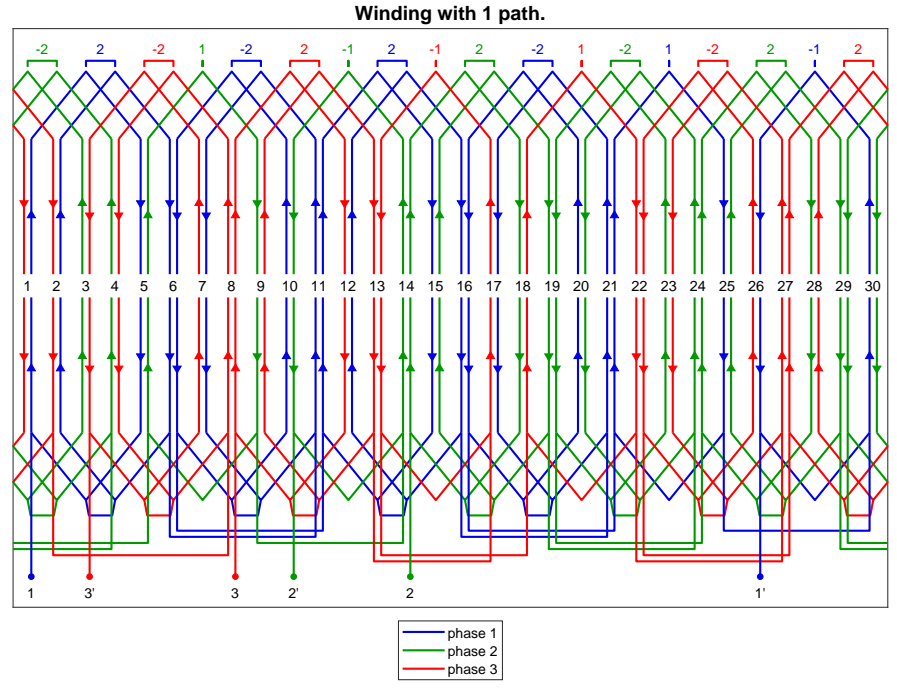

(a) Winding scheme.

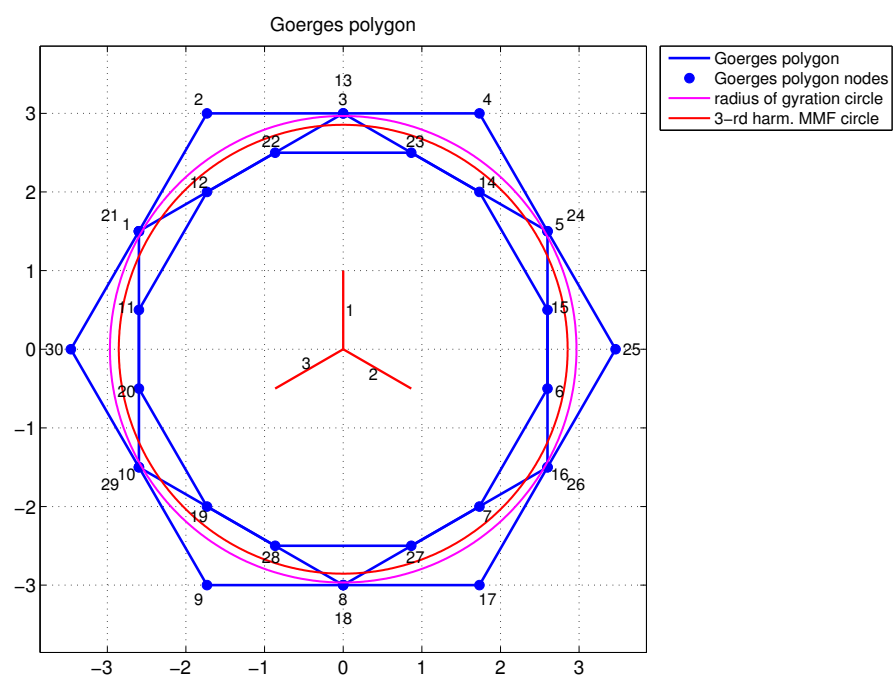

(b) Görges polygon.

Figure 7. 3-phase machine with asymmetrical double-layer fractional winding: $N=30, p=3, \eta=0$.

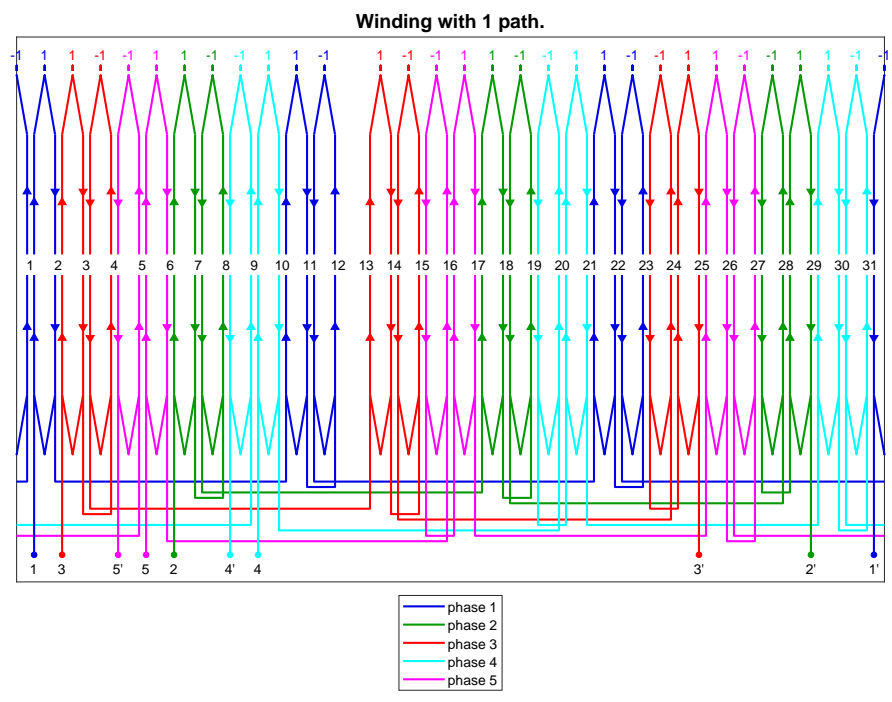

(a) Winding scheme.

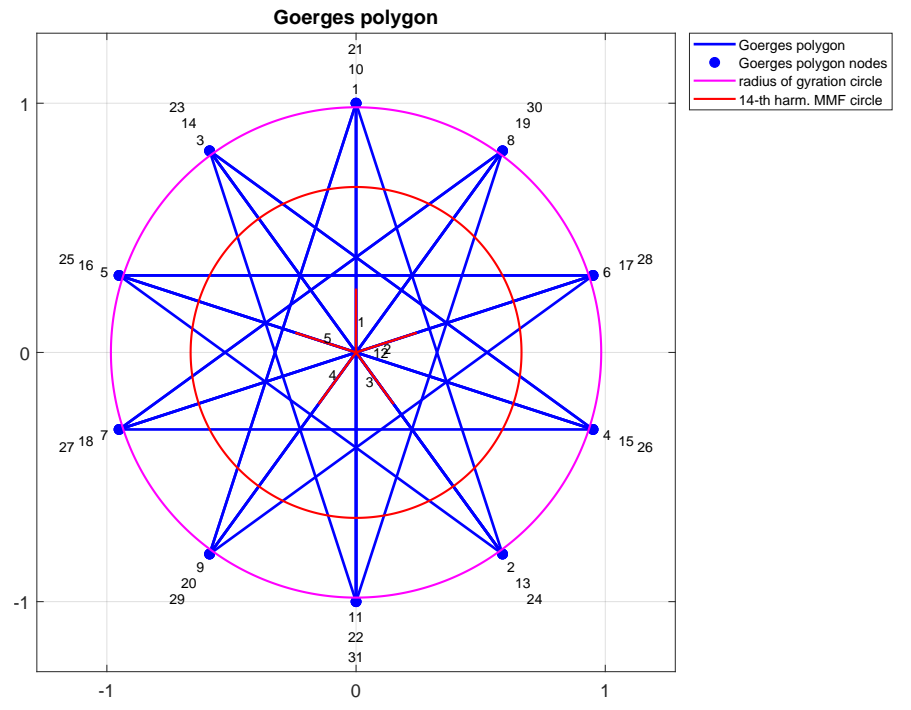

(b) Görges polygon.

Figure 8. 5-phase machine with asymmetrical double-layer fractional winding: $N=31, p=14, \eta=1$.

\section{REFERENCES}

$$
\begin{gathered}
\sigma_{0 m}=\frac{J_{p}}{N \cdot V_{p}^{2}}-1=\frac{3.6426+3.2143}{7 \cdot 0.9241^{2}}-1=0.1471, \\
\sigma_{0 v}=\frac{2}{7 \cdot 0.924^{2}} \sqrt{\left(\frac{3.6426-3.2143}{2}\right)^{2}+0.3711^{2}}=0.1433 .
\end{gathered}
$$

\section{ACKNOWLEDGMENTS}

This work was financially supported by MIUR, Ministero dell'Istruzione dell'Universita' e della Ricerca (Italian Ministry of Education, University and Research) and by SDESLab (Sustainable Development and Energy Saving Laboratory) of the University of Palermo.
[1] G. Müller and B. Ponick, Theorie elektrischer Maschinen. Elektrische Maschinen, Wiley-VCH Verlag GmbH \& Co KGaA, 6 ed., 2009.

[2] G. Müller, K. Vogt, and B. Ponick, Berechnung elektrischer Maschinen. Elektrische Maschinen, Wiley-VCH Verlag GmbH \& Co KGaA, 6 ed. 2008.

[3] D. Kocabas, "Novel Winding and Core Design for Maximum Reduction of Harmonic Magnetomotive Force in AC Motors," IEEE Transactions on Magnetics, vol. 45, pp. 735-746, Feb 2009.

[4] J. Bacher, H. Köfler, and G. Maier, "The Use of Pitch Factor in Calculations of AC-machines with Concentrated Windings," in EPE 2005, pp. 1-6, European Power Electronics and Drives Association, 2005.

[5] J. Steinbrink, "Design and Analysis of Windings of Electrical Machines," in International Symposium on Power Electronics, Electrical Drives, Automation and Motion, 2008. SPEEDAM 2008, pp. 717-720, June 2008.

[6] A. Smith and D. Delgado, "Automated AC Winding Design," in 5th IET International Conference on Power Electronics, Machines and Drives (PEMD 2010), pp. 1-6, April 2010.

[7] A. EL-Refaie, "Fractional-Slot Concentrated-Windings Synchronous Permanent Magnet Machines: Opportunities and Challenges," IEEE Transactions on Industrial Electronics, vol. 57, pp. 107-121, Jan 2010. 


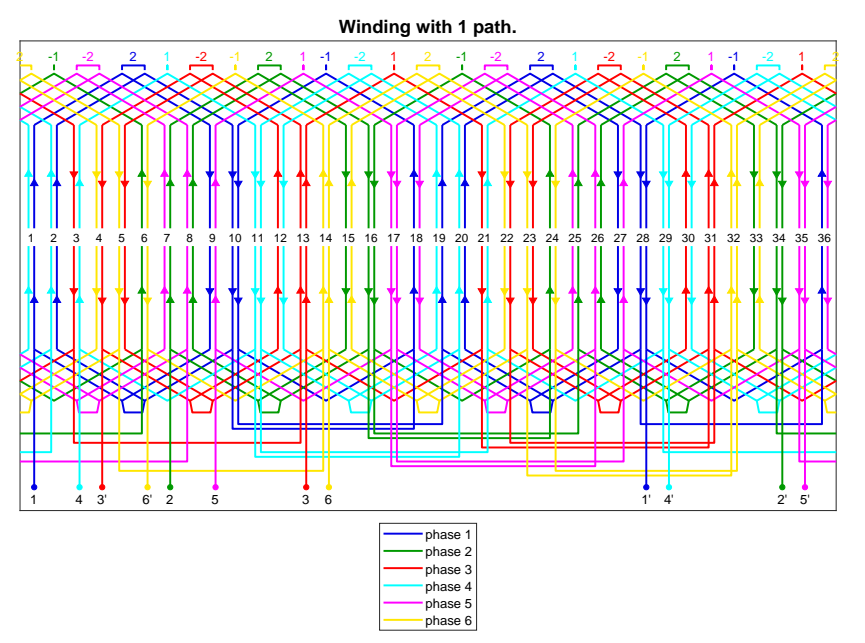

(a) Winding scheme.

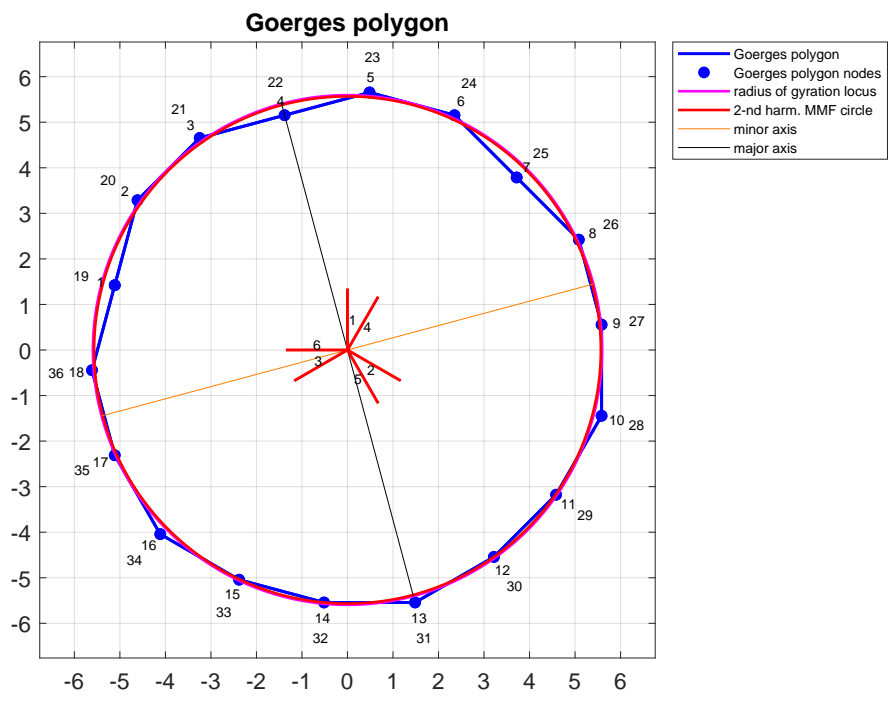

(c) Görges polygon.
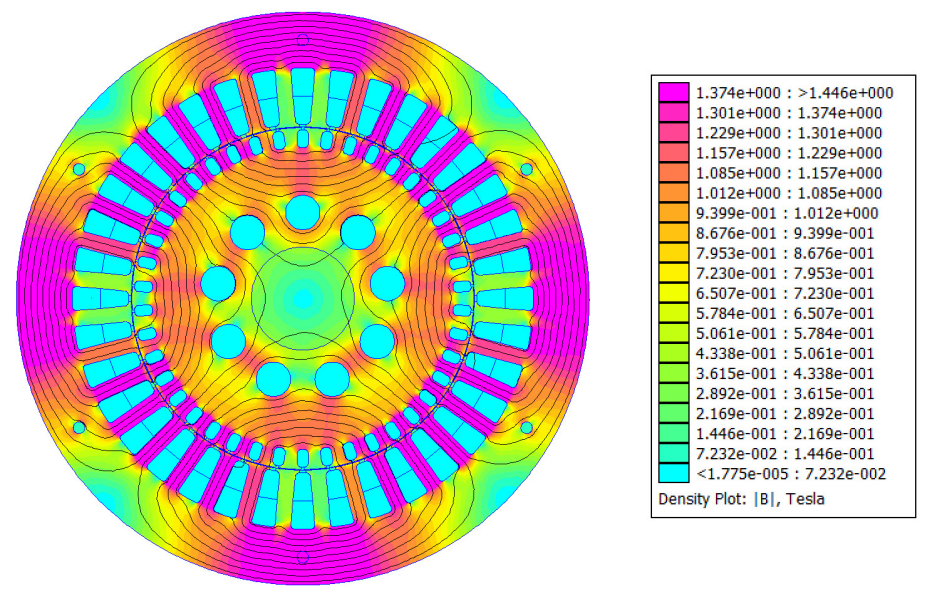

(b) Simulation results.

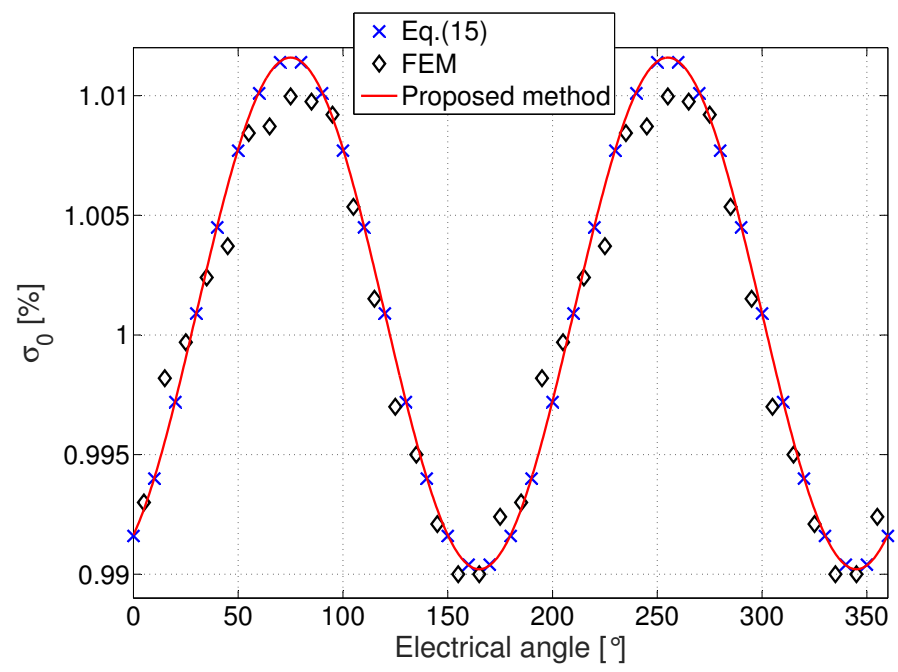

(d) FEM validation.

Figure 9. FEM validation for a 6-phase machine with a reduced symmetrical double-layer winding: $N=36, p=2$.

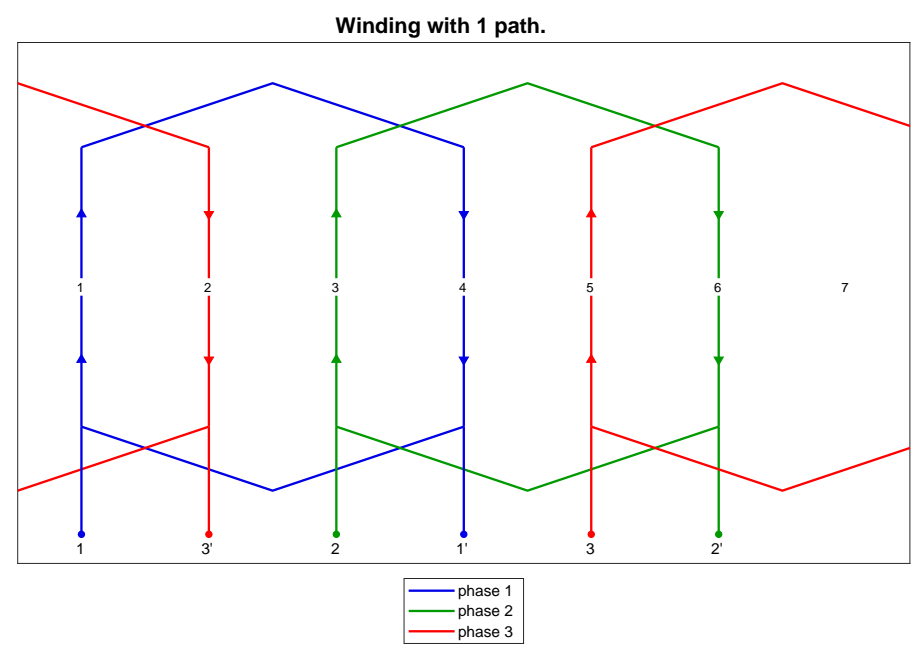

Figure 10. Winding scheme of the proposed example.

[8] A. Zavvos, A. McDonald, and M. Mueller, "Optimisation tools for large permanent magnet generators for direct drive wind turbines," Renewable Power Generation, IET, vol. 7, pp. 163-171, March 2013.
[9] Z. Zhu and X. Liu, "Novel stator electrically field excited synchronous machines without rare-earth magnet," IEEE Transactions on Magnetics, vol. 51, pp. 1-9, April 2015.

[10] I. Boldea and S. Nasar, The Induction Machine Handbook. Electric Power Engineering Series, CRC Press, 2010.

[11] H. Xueliang, D. Qiang, and H. Minqiang, "A novel exact and universal approach for calculating the differential leakage related to harmonic waves in ac electric motors," IEEE Transactions on Energy Conversion, vol. 19, pp. 1-6, March 2004.

[12] B. Heller and V. Hamata, Harmonic field effects in induction machines. Elsevier Scientific Pub. Co., 1977.

[13] R. Richter, Die Induktionsmaschinen, vol. 4 of Elektrische Maschinen. Basel, Stuttgart: Birkhäuser, 2 ed., 1954.

[14] H. Sequenz, Die Wicklungen elektrischer Maschinen: WechselstromSonderwicklungen, vol. 3. Wien, Austria: Springer Verlag, 1954.

[15] M. M. Liwschitz, "Differential leakage of the different patterns of a fractional slot winding," Transactions of the American Institute of Electrical Engineers, vol. 68, pp. 1129-1132, July 1949.

[16] M. M. Liwschitz, "Differential leakage of a fractional-slot winding," Electrical Engineering, vol. 65, pp. 314-320, May 1946.

[17] M. M. Liwschitz, "Differential leakage with respect to the fundamental wave and to the harmonics," Electrical Engineering, vol. 63, pp. 11391149, Dec 1944.

[18] F. Heller and W. Kauders, "Das Görgessche Durchflutungspolygon," Archiv für Elektrotechnik, vol. 29, no. 9, pp. 599-616, 1935.

[19] D. Hiramatsu, K. Sutrisna, H. Ishizuka, M. Okubo, K. Tsujikawa, T. Ueda, H. Hachiya, J. Mori, T. Aso, and T. Otaka, "Study of Winding Method to Reduce Stray Loss and Stator Core Vibration of Synchronous Machine," 
in 2013 International Conference on Electrical Machines and Systems (ICEMS), pp. 36-41, Oct 2013.

[20] M. Caruso, A. O. Di Tommaso, R. Miceli, and R. Rizzo, "The use of slightly asymmetrical windings for rotating electrical machines," International Transactions on Electrical Energy Systems, no. e2579, 2018.

[21] L. Melcescu, M. Cistelecan, O. Craiu, and H. Cosan, "A new 4/6 polechanging double layer winding for three phase electrical machines," in 2010 XIX International Conference on Electrical Machines (ICEM), pp. 16, Sept 2010.

[22] M. V. Cistelecan, B. Cosan, and M. D. Popescu, "Analysis and Design Criteria for Fractional Unbalanced Windings of Three-phase Motors," in 6th International Symposium on Advanced Electromechanical Motion Systems - ELECTROMOTION 2005, pp. 1-5, September 2005.

[23] R. Hellmund and C. Veinott, "Irregular windings in wound rotor induction motors," Electrical Engineering, vol. 53, pp. 342-346, Feb 1934.

[24] H. Sequenz, Die Wicklungen elektrischer Maschinen: Wechselstrom Ankerwicklungen., vol. 1. Wien, Austria: Springer Verlag, 1950.

[25] C. Lu, S. Ferrari, and G. Pellegrino, "Two design procedures for pm synchronous machines for electric powertrains," IEEE Transactions on Transportation Electrification, vol. 3, pp. 98-107, March 2017.

[26] G. Huth, "Optimierung des Wicklungssystems bei permanentmagneterregten AC - Servomotoren," Electrical Engineering, vol. 8, pp. 375-383, 1999.

[27] A. O. Di Tommaso, F. Genduso, R. Miceli, and G. Ricco Galluzzo, "An exact method for the determination of differential leakage factors in electrical machines with non-symmetrical windings," IEEE Transactions on Magnetics, vol. 52, pp. 1-9, Sept 2016.

[28] J. Pyrhonen, T. Jokinen, and V. Hrabovcová, Design of rotating electrical machines. New Delhi, India: John Wiley \& Sons, 2009.

[29] M. Caruso, A. O. Di Tommaso, F. Marignetti, R. Miceli, and G. Ricco Galluzzo, "A general mathematical formulation for winding layout arrangement of electrical machines," Energies, vol. 11, no. 2, 2018.

[30] K. Hruska and M. Sokol, "Advanced numeric tooth winding analyses," in 2012 15th International Symposium MECHATRONIKA, pp. 1-7, Dec 2012.

[31] A. Kron and K. Bopp, "Beitrag zur praktischen Berechnung des Koeffizienten der doppeltverketteten Streuung," Archiv für Elektrotechnik, vol. 41, no. 3, pp. 136-142, 1953.

[32] M. Cistelecan, L. Melcescu, H. Cosan, and M. Popescu, "Induction motors with changeable pole windings in the ratio 1:4," in 2011 International Aegean Conference on Electrical Machines and Power Electronics and 2011 Electromotion Joint Conference (ACEMP), pp. 781-786, Sept 2011.

[33] F. W. Carter, "The magnetic field of the dynamo-electric machine," Electrical Engineers, Journal of the Institution of, vol. 64, pp. 1115-1138, November 1926.

[34] R. Richter, Allgemeine Berechunungselemente - Gleichstrommaschinen, vol. 1 of Elektrische Maschinen. Basel, Stuttgart: Birkhäuser, 3 ed., 1967.

[35] C. L. Fortescue, "Method of symmetrical co-ordinates applied to the solution of polyphase networks," Proceedings of the American Institute of Electrical Engineers, vol. 37, pp. 629-716, June 1918.

[36] A. O. Di Tommaso, F. Genduso, R. Miceli, and G. Ricco Galluzzo, "Determination of differential leakage factors in electrical machines with nonsymmetrical full and dead-coil windings," in 2017 Twelfth International Conference on Ecological Vehicles and Renewable Energies (EVER), pp. 1-6, April 2017.

[37] A. O. Di Tommaso, F. Genduso, and R. Miceli, "A new software tool for design, optimization, and complete analysis of rotating electrical machines windings," IEEE Transactions on Magnetics, vol. 51, pp. 1-10, April 2015

[38] M. Caruso, A. O. Di Tommaso, R. Miceli, and R. Rizzo, "Computer-aided analysis and design procedure for rotating induction machine magnetic circuits and windings," IET Electric Power Applications, vol. 12, no. 6, pp. 885-893, 2018.

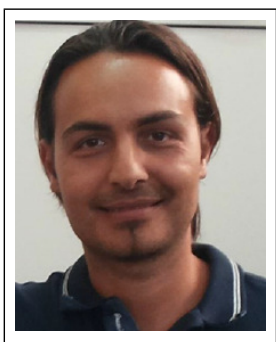

Massimo Caruso received the M.S. and Ph.D. degrees in electrical engineering from the University of Palermo, Palermo, Italy, in 2008 and 2012, respectively. In 2011, he joined the MEMS Sensors and Actuators Laboratory Group, University of Maryland, College Park, MD, USA, collaborating for the development of microsensors for in vivo bacteria biofilm detection and treatment. In 2014, he joined the Sustainable Development and Energy Saving Laboratory, University of Palermo, Palermo, Italy, focusing his research activity on electrical machines, power electronics and electrical drives.

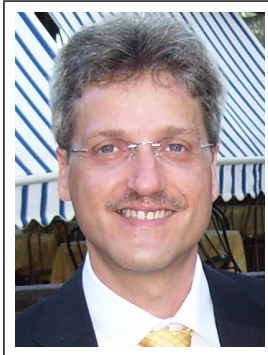

Antonino Oscar Di Tommaso received the M.S and $\mathrm{Ph} . \mathrm{D}$. degrees in electrical engineering from the University of Palermo, Italy, in 1999 and 2004, respectively. He was a Post Ph.D. Fellow of Electrical Machines and Drives with the Department of Electrical Engineering, University of Palermo, from 2004 to 2006. He is currently a Researcher and Assistant Professor of Electrical Machines with the Department of Energy, Information engineering and Mathematical models (DEIM), University of Palermo. His research interests include electrical machines, drives, diagnostics on power converters, and diagnostics and design of electrical rotating machines.

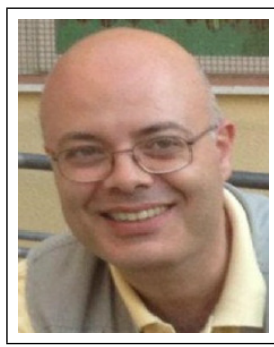

Fabio Genduso received the M.S. and Ph.D. degrees in electrical engineering from the University of Palermo, Palermo, Italy, in 1999 and 2004, respectively. In 2005, he joined the Department of Energy, Information engineering and Mathematical models (DEIM), University of Palermo, as a Post Ph.D. Fellow and an Assistant Professor. He taught power electronics and drives, electrical machines, network theory, and control of electrical drives in many undergraduate and post graduate courses. He is a DEIM Researcher and the responsible of the research unit on electrical drives and power converters within the Sustainable Development and Energy Saving Laboratory (SDESLAB), University of Palermo. His research interests include power electronics, electrical drives and control, power system control, microgrids, and solar and wind energy.

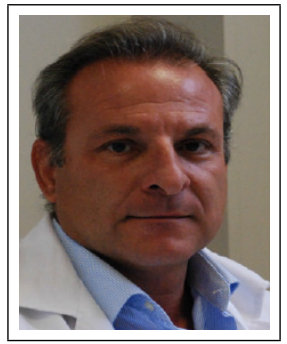

Rosario Miceli Rosario Miceli (M'01) received the B.S. degree in electrical engineering and the Ph.D. degree from the University of Palermo, Palermo, Italy, in 1982 and 1987, respectively. He is a Full Professor of electrical machines with the Polytechnic School, University of Palermo. He is a Personnel in Charge of the Sustainable Development and Energy Savings Laboratory of the University of Palermo. His main research interests include mathematical models of electrical machines, drive system control, and diagnostics, renewable energies, and energy management. Dr. Miceli is a Reviewer for the IEEE Transactions on Industrial Electronics and the IEEE Transactions on Industry Applications.

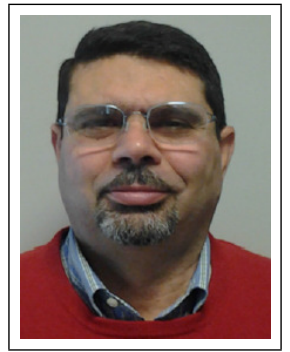

Giuseppe Ricco Galluzzo received the B.S. degree in electrical engineering from the University of Palermo, Palermo, Italy, in 1978. From 1992 to 2001, he was an Associate Professor of Electrical Drives with the University of Palermo, where, since September 2001, he is a Full Professor of Electrical Drives. His research interests include mathematical models of electrical machines and drive-system control and diagnostics. 